\title{
Restriction of the activity of the recombination site dif to a small zone of the Escherichia coli chromosome
}

\author{
François Cornet, Jacqueline Louarn, Josette Patte, and Jean-Michel Louarn ${ }^{1}$ \\ Laboratoire de Microbiologie et de Génétique Moléculaire, Centre National de la Recherche Scientifique, 31062 Toulouse \\ Cedex, France
}

\begin{abstract}
The recombination site dif is the target on the Escherichia coli chromosome of the site-specific recombinases $\mathrm{XerC}$ and XerD. The dif/XerC-D system plays a role during the cell cycle, probably by favoring sister chromosome monomerization or separation. A phenomenon of regional control over dif activity, also analyzed in this issue, is demonstrated here by translocation of dif to a series of loci close to the normal locus. We found that the site is physiologically active only within a narrow zone around its natural position. Competence for dif activity does not depend on the sequence of the normal dif activity zone (DAZ), because $\Delta($ dif $)$ deletions larger than the DAZ result in Dif $^{+}$bacteria when dif is reinserted at the junction point. Although dif maps where replication normally terminates, termination of replication is not the elicitor. A strain with a large inversion that places $d i f$ and its surrounding region close to oriC remains $\mathrm{Dif}^{+}$, even when a Tus ${ }^{-}$mutation allows replication to terminate far away from it. Preliminary data suggest the possibility that specialized sequences separate the competent zone from the rest of the chromosome. We suspect that these sequences are members of a set of sequences involved in a polarized process of postreplicative reconstruction of the nucleoid structure. We propose that this reconstruction forces catenation links between sister chromosomes to accumulate within the DAZ, where they eventually favor recombination at dif.
\end{abstract}

[Key Words: Site-specific recombination; dif site domain of activity; E. coli chromosome organization]

Received January 10, 1996; revised version accepted March 20, 1996.

The Escherichia coli chromosome harbors a recombination site, dif, that maps where replication normally terminates. Bacteria deficient for dif-specific recombination display decreased growth rate and colony forming ability and frequently form filaments with nucleoid partitioning defects (Blakely et al. 1991, 1993; Kuempel et al. 1991). Recombination between two dif sites is catalyzed by the XerC and XerD recombinases, which are also involved in the resolution of plasmid multimers (Blakely et al. 1991, 1993). It has been proposed that dif facilitates chromosome separation before partition, for instance, by resolving chromosome dimers that result from an odd number of exchanges between sister chromosomes (Blakely et al. 1991; Kuempel et al. 1991). This physiological role may involve frequent exchanges between dif sites belonging to sister chromosomes, fused or not. Because dif lies in the region of the chromosome replicated last (Louarn et al. 1994), a pair of dif sites (the normal target for a recombinase) is available only at termination. Consequently, site-specific recombination at dif should proceed most often between complete sister chromosomes. This implies that the spatial positioning inside the cell of two dif regions belonging to different

\footnotetext{
${ }^{1}$ Corresponding author.
}

nucleoids should at least create no obstacle to, and perhaps could facilitate, exchanges between the sites.

The location of the dif site on the chromosome seems critical for its activity. A Dif ${ }^{-}$phenotype results from translocations of dif to the lac operon at $8 \mathrm{~min}$ on the chromosome map, to a site near oriC (Leslie and Sherratt 1995), and even to a site within the terminus region, 118 $\mathrm{kb}$ to the left of its natural position (Tecklenburg et al. 1995). Nevertheless, these translocated sites were still able to recombine with a plasmid-borne dif site. Conversely, replacing dif at its natural position by either $p s i$, the resolution site of plasmid pSCl01, or $\operatorname{lox} P$, the resolution site of bacteriophage Pl (in the presence of Cre resolvase), does not disrupt the Dif ${ }^{+}$phenotype (Cornet et al. 1994; Leslie and Sherratt 1995). Therefore, the location of the recombination site seems more important for the chromosome than the nature of the site itself. Another remarkable feature of the region containing dif is a high frequency of excision by homologous recombination between direct repeats (Louarn et al. 1991). This phenomenon, called terminal recombination, is independent of termination of replication and of any unique sequence of the region, and is probably caused by interchromosomal exchanges (Louarn et al. 1994; J. Louarn, F. Cornet, J. Patte, and J.M. Louarn, unpubl.). Although difspecific and excisive recombination processes are mech- 
anistically unrelated, they share the property of being elicited in the same chromosomal region. Whether the two recombination processes are under the same regional control and how this control is exerted, are the two major questions investigated in this paper.

Our data indicate that the competent zone for dif activity is restricted to a small region around its natural position, confirming the observations presented by Kuempel et al. (this issue). Deletion analyses indicate that no specific sequence-eliciting dif activity exists within the competent zone, thus showing that competence for dif activity is created by external factors. In addition, we demonstrate that replication termination does not determine the location of the competent zone. We have also begun to analyze the region of transition between the competent zone and the rest of the chromosome. The data suggest that the transition effect is mediated by specific sequences.

\section{Results}

The dif translocation system and criteria for dif activity

Most of the experiments are based on relocating dif by deleting a short fragment containing the dif site and then reinserting a dif-containing fragment at predetermined chromosomal positions. The net result is a translocation of the dif site. In all constructions, the final translocation consists of the insertion of a 1.6-kb chromosomal dif fragment into the EcoRV restriction site of a tet $A$ gene belonging to either a $\operatorname{Tn} 10$ transposon or a $\operatorname{Tn} 10$ subfragment inserted previously at a desired position into the chromosome of $\Delta($ dif $)$ strain LN3061. An example of the genetic structures involved is shown in Figure 1. These constructions involved several steps of sequence replacements which were effected using an integration-excision vector described earlier (Cornet et al. 1994). The recombinant plasmids thus constructed are described in Table 1.

The physiological activity of a translocated dif site cannot be measured by a simple test reaction. Five characteristics differentiate a $\Delta($ dif $)$ strain from its wild-type counterpart: filamentation, slower growth rate, lower colony forming efficiency, induction of the SOS system, and low recombination between a dif-carrier plasmid and the chromosome (Kuempel et al. 1991; this work). All of these characteristics except SOS induction have been measured in this study. High frequency of dif-carrier plasmid integration was observed at all translocated dif sites (see Tables 2-4), indicating that all of these sites can undergo XerCD-catalyzed recombination. Although the presence of filaments is a sensitive criterium, their frequencies are somewhat variable and not easily quantified, as also noticed by Tecklenburg et al. (1995). The activity of a translocated dif site was in consequence evaluated by measuring growth rate and/or colony-forming ability in addition to microscopic observation. The site was taken to be physiologically inactive (Dif ${ }^{-}$phenotype) when generation times and colony-forming abil-
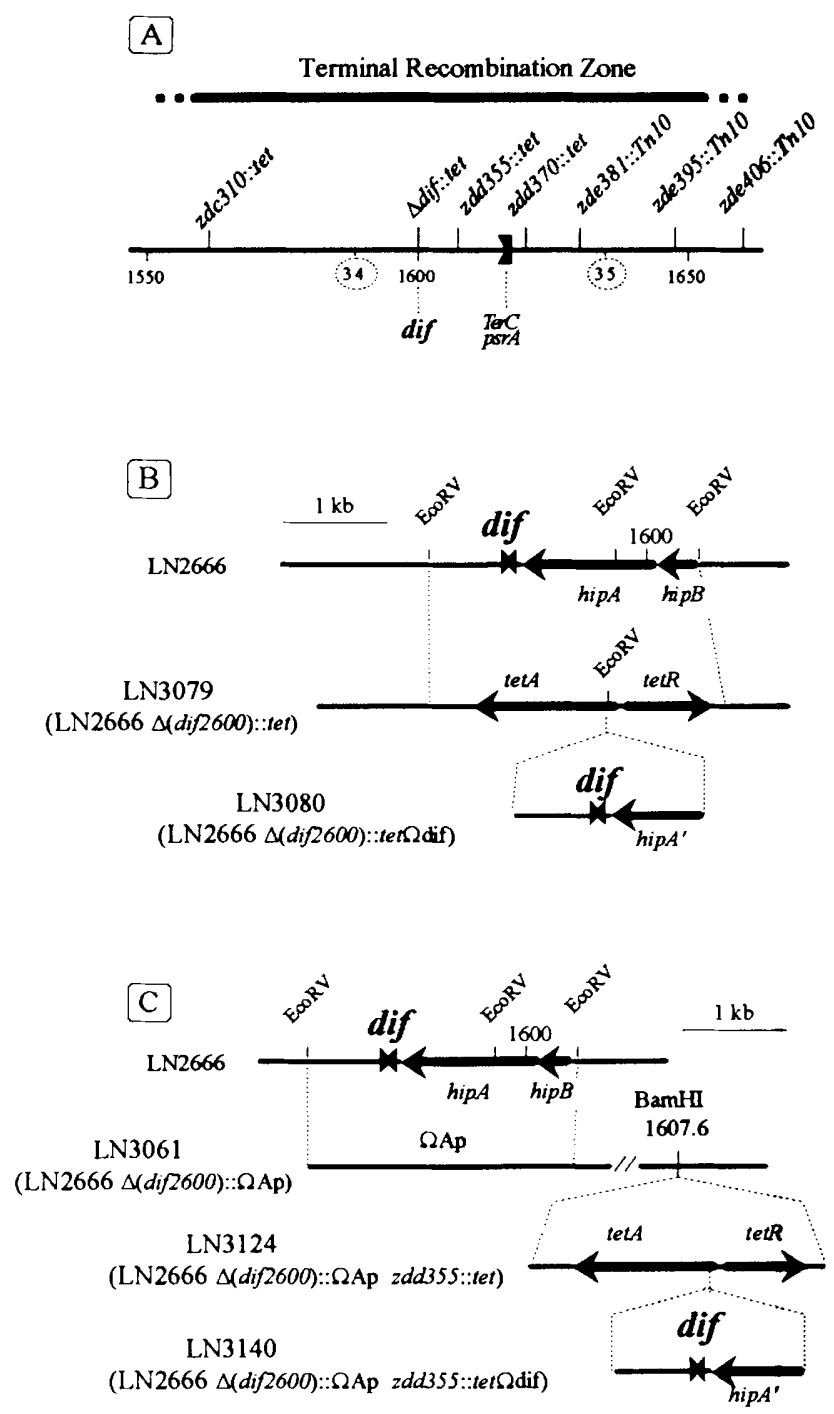

Figure 1. The dif translocation system. (A) Map of the dif region on the $E$. coli chromosome. Coordinates are given in $\mathrm{kb}$ and min (numbers in dotted circles) according to Rudd's map (K. Rudd, pers. comm.l. The locations of the Tn10 and tet insertions used, as well as the size of the region where terminal recombination has been observed (Louarn et al. 1994; J. Louarn, F. Cornet, J. Patte, and J.M. Louarn, unpubl.), are indicated. (B) Two-step construction of the $J($ dif2600)::tet $\Omega$ dif strain. The cloned dif chromosomal fragment has been modified by deletion of dif and insertion of tet in vitro (plasmid pFC75 of Table 1) and then recombined into the chromosome to yield strain LN3079. The 1.6-kb dif-containing fragment was eventually crossed into the chromosomal tet $A$ gene using the delivery plasmid pFC72 as described in Materials and methods. $(C)$ Construction and typical structure of a strain harboring a translocated dif site. The starting strain is LN3061, which harbors

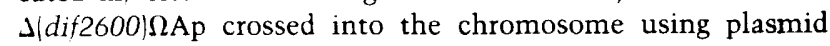
pFC73 (Table 1). The tet segments inserted at various positions along the chromosome (the zdd355::tet insertion shown here) were crossed into this strain by $\mathrm{Pl}$ transduction. The dif site was eventually inserted into the tet $A$ locus using $\mathrm{pFC72}$ 
Table 1. Plasmids used

\begin{tabular}{|c|c|}
\hline Plasmid & Description and References ${ }^{a}$ \\
\hline $\mathrm{C} 9$ & g a synthetic 33-bp dif site (Cornet et al. 1994). \\
\hline pFC20 & an integration-excision vector used for substitution of chromosomal fragments /Cornet et al. 1 \\
\hline pLN135 & pFC20 with a modified polylinker harboring Nsil and HindIII sites ${ }^{\mathrm{b}}$ \\
\hline pLN138 & $\begin{array}{l}\text { pLN135 carrying the 1561.4-1565.8 PstI-KpnI chromosomal fragment from pBS10 (Béjar and Bouché 1983) cloned in } \\
\text { the polylinker }\end{array}$ \\
\hline pFC68 & $\begin{array}{l}\text { pFC20 with the } 2.8-\mathrm{kb} \text { Bst YI tet fragment from Tn10 (from pFC35; Cornet et al. 1994) inserted into the BamHI site of } \\
\text { the polylinker. }\end{array}$ \\
\hline pLN139 & $\begin{array}{l}\text { pLN138 carrying the } 2.8-\mathrm{kb} \text { Bst YI tet fragment from pFC68 cloned in the BamHI site creating the } z d c 310:: \text { tet } \\
\text { insertion at position } 1563.0 \text {. }\end{array}$ \\
\hline $\mathrm{pFC72}$ & $\begin{array}{l}\text { pFC68 carrying the } 1597.8-1599.8 \text { EcoRV dif-containing chromosomal fragment (from pBS12; Béjar and Bouché } 1983 \text { ) } \\
\text { cloned into the EcoRV site of the tetA gene. The two orientations of dif with respect to tet } A \text { exist (see Material and } \\
\text { methods, Fig. 1). }\end{array}$ \\
\hline pFC73 & 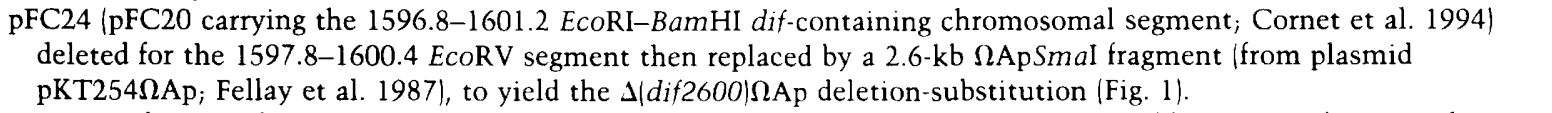 \\
\hline pFC75 & $\begin{array}{l}\text { pFC73 with a } 2.6-\mathrm{kb} \text { HindIII deletion removing the } \Omega \text { Ap interposon, replaced by the } 2.8-\mathrm{kb} \text { Bst YI tet fragment from } \\
\text { pFC68, to yield the } \Delta(\text { dif2600)::tet deletion-substitution. }\end{array}$ \\
\hline pFC85 & $\begin{array}{l}\text { pLN135 carrying the 1603.7-1611.7 KpnI chromosomal fragment from pBS12 (Béjar and Bouché 1983) cloned in the } \\
\text { polylinker. }\end{array}$ \\
\hline pFC86 & $\begin{array}{l}\text { pFC } 85 \text { with the } 2.8-\mathrm{kb} \text { Bst YI tet fragment from pFC } 68 \text { cloned in the BamHi site at position } 1607.6 \text {, creating the } \\
\text { zdd355::tet insertion. }\end{array}$ \\
\hline pFC89 & $\begin{array}{l}\text { pLN135 with the } 1620.3-1623.6 \mathrm{KpnI}-\mathrm{Bg} / \mathrm{I} \text { chromosomal fragment from Kohara phage } 304 \text { (Kohara et al. 1987) cloned } \\
\text { into the polylinker }{ }^{\mathrm{C}}\end{array}$ \\
\hline pFC90 & $\begin{array}{l}\text { pFC89 with the } 2.8 \mathrm{~kb} \text { BstYI tet fragment from pFC68 inserted in the BamHI site at position } 1622.4 \text {, creating the } \\
z d d 370:: \text { tet insertions at position } 1622.4 \text { on Rudd's map. }\end{array}$ \\
\hline pFC91 & $\begin{array}{l}\text { pFC } 89 \text { deleted of the } 1620.3-1622.4 \mathrm{KpnI}-\mathrm{BamHI} \text { segment then replaced by the } 1603.7-1607.6 \mathrm{KpnI}-\mathrm{BamH} \text { fragment } \\
\text { from pFC85, to yield the } \Delta(z d d 355-z d d 370) \text { deletion. }\end{array}$ \\
\hline pFC92 & 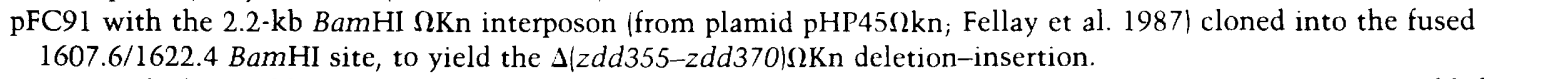 \\
\hline pFC93 & $\begin{array}{l}\text { pFC91 with the } 2.8-\mathrm{kb} \text { BstYI tet fragment from pFC68 cloned into the fused } 1607.6 / 1622.4 \text { Bam HI site, to yield the } \\
\Delta(z d d 355-z d d 370):: \text { tet deletion-insertion. }\end{array}$ \\
\hline pFC94 & $\begin{array}{l}\text { pLN135 carrying a } 20-\mathrm{kb} \text { chromosomal fragment }(1603.7-1623.6) \text { obtained by in vivo recombination between pFC } 92 \\
\text { and the E. coli chromosome (detailed in Materials and methods). }\end{array}$ \\
\hline pFC95 & $\begin{array}{l}\text { pFC94 deleted for a } 4.6-\mathrm{kb} B F / \mathrm{II} \text { chromosomal segment then replaced by the } 2.2-\mathrm{kb} \text { BamHI } \Omega \mathrm{Kn} \text { interposon, to yield } \\
\text { the } \Delta(1612.2-1616.8) \Omega \mathrm{Kn} \text { deletion-insertion. }\end{array}$ \\
\hline pFC97 & 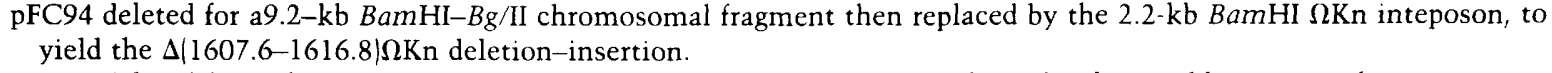 \\
\hline pFC101 & $\begin{array}{l}\text { pFC94 deleted for a chromosomal } 10.1-\mathrm{kb} \text { Bam HI-BglII segment then replaced by the } 2.8-\mathrm{kb} \text { Bst YI tet fragment, to } \\
\text { yield the } \Delta(1612.2-1622.4): \text { tet deletion-insertion. }\end{array}$ \\
\hline 102 & $\begin{array}{l}\text { pFC94 deleted for a chromosomal } 9.2-\mathrm{kb} \text { BamHI-BglII segment then replaced by the } 2.8-\mathrm{kb} \text { Bst YI tet fragment, to } \\
\text { yield the } \Delta(1607.6-1616.8): \text { :tet deletion-insertion. }\end{array}$ \\
\hline $\mathrm{pFC}$ & $\begin{array}{l}\text { pFC94 deleted for a chromosomal } 4.6-\mathrm{kb} \text { BglII fragment then replaced by the } 2.8-\mathrm{kb} \text { Bst YI tet fragment, to yield the } \\
\Delta(1612.2-1616.8): \text { tet deletion-insertion. }\end{array}$ \\
\hline
\end{tabular}

${ }^{2}$ For sake of identification, chromosomal restriction site ordinates refer to the Ecomap6 of $\mathrm{K}$. Rudd (pers. comm.). 'The two complementary oligonucleotides 5'-GATCATGCATGAAGCTT-3' and 5'-GATCAAGCTTCATGCAT-3' were mixed in TE buffer (Sambrook et al. 1989) at $6.25 \mu \mathrm{g} / \mathrm{ml}$, heated at $94^{\circ} \mathrm{C}$ for $2 \mathrm{~min}$, and cooled slowly to $4^{\circ} \mathrm{C}$ in $5 \mathrm{hr}$. The annealed oligonucleotides were then ligated of pFC20 digested with BamHI.

${ }^{\mathrm{c}} \mathrm{A} 7.6-\mathrm{kb}$ KpnI fragment from Kohara phage $\lambda 304$ was first cloned in the KpnI site of pLN135. The resultant plasmid pFC 88 was digested with HindIII and BgII to remove a 4.3-kb fragment. Ends were blunted using T4 DNA polymerase (Sambrook et al. 1989), and the resulting fragment was self-ligated.

ities were similar to that of a reference $\Delta($ dif $)$ strain $\{34$ $36 \mathrm{~min}$ and $\sim 65 \%$, respectively, in Luria-Bertani medium (LB) at $37^{\circ} \mathrm{C}$, compared with $25-26 \mathrm{~min}$ and $>99 \%$ for the wild-type strain LN2666 in the same conditions).

The dif translocation system has been validated by showing that insertion of the dif-carrying $1.6-\mathrm{kb}$ fragment into the tet determinant of a $\Delta($ dif2600)::tet deletion (LN3080 in Fig. 1) largely restores the Dif ${ }^{+}$pheno- type, that is, normal generation time (Table 2, below), considerable improvement of the efficiency of microcolony formation (Fig. 4, below), and strong reduction of filamentation despite a cell-size dispersion larger than in the Dif $^{+}$parent (data not shown). Because all strains with a translocated dif site have undergone the $\Delta($ dif2600) deletion and harbor the same dif fragment inserted into the tet segment, as in LN3080, the behavior 
Table 2. Expression of the Dif phenotype in various dif translocations

\begin{tabular}{|c|c|c|c|c|c|c|}
\hline Strain & Relevant genotype & Filaments & $\begin{array}{l}\text { Doubling } \\
\text { time }\end{array}$ & $\begin{array}{l}\text { Efficiency of } \\
\text { plating }|\%|\end{array}$ & $\begin{array}{l}\text { Integration } \\
\text { of } \mathrm{pFC} 9\end{array}$ & $\begin{array}{l}\text { Dif } \\
\text { phenotype }\end{array}$ \\
\hline LN2666 & $1+1$ & A & 25 & $>99$ & $6.410^{-2}$ & + \\
\hline LN3079 & $\Delta($ dif2600)::tet & $\mathrm{F}$ & 36 & 60 & $2.310^{-5}$ & - \\
\hline LN3080 & $\Delta(\operatorname{dif} 2600)::$ tet $\Omega$ dif & A & 25 & 96 & $9.610^{-2}$ & + \\
\hline LN3061 & $\Delta($ dif2600 $) \Omega A p$ & $\mathrm{~F}$ & 35 & 65 & $1.110^{-4}$ & - \\
\hline LN3320 & LN3061 zdc310::tet $\Omega$ dif & $\mathrm{F}$ & 35 & N.D. & $5.010^{-2}$ & - \\
\hline LN3161 & LN3061 zdd355::tet $\Omega$ dif & $\mathrm{R}$ & 26 & 88 & $1.710^{-1}$ & + \\
\hline LN3162 & LN3061 zdd370::tet $\Omega$ dif & $\mathrm{F}$ & 36 & 60 & $8.110^{-2}$ & - \\
\hline LN3046 & 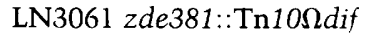 & $\mathrm{F}$ & 35 & N.D. & $6.110^{-2}$ & - \\
\hline LN3093 & 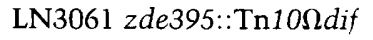 & $\mathrm{F}$ & 37 & N.D. & $5.910^{-2}$ & - \\
\hline LN3092 & 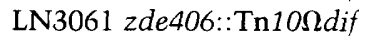 & $\mathrm{F}$ & 37 & N.D. & $6.210^{-2}$ & - \\
\hline
\end{tabular}

The general procedures for strain construction are described in Materials and methods and Fig. 1. Plasmids used for dif translocation are listed in Table 1. Filamentation was observed by phase-contrast microscopy: (F) Frequent filaments, as in $\Delta($ dif $)$ strain LN3061: (R) rare filaments; (A) absence of filaments. Doubling times (in min, averages of two determinations) were measured in standardized conditions of exponential growth in LB medium at $37^{\circ} \mathrm{C}$. Percentages of bacteria spread on agar slides giving rise to microcolonies were determined as described in Materials and methods; values indicated are the averages of several determinations, corresponding to the fate of several thousands bacterial cells. Integration frequencies of pFC9 are the means of four determinations of the ratio of colonyforming units at $42^{\circ} \mathrm{C}$ to those at $30^{\circ} \mathrm{C}$ on streptomycin plates. (N.D.) Not done.

of this strain is taken as the reference. Importantly, we found throughout this study that regardless of the location of dif, the orientation of the dif-containing fragment in the chromosome did not affect the Dif phenotype (data not shown).

\section{Size of the zone competent for dif activity}

We have translocated dif into six sites within the terminus region (Fig. 1). One translocation site mapped $37 \mathrm{~kb}$ to the left of dif, into $z d c 310:$ :tet (LN3320), and the five others to its right, $z d d 355::$ tet (LN3161), zdd370:: tet (LN3162), zde381::Tn10 (LN3046), zde395::Tn10 (LN3093), zde406::Tn10 (LN3092), at 8, 23, 34, 48, and $59 \mathrm{~kb}$, respectively, from dif. These insertions have no effect on growth rates or on efficiency of colony formation when introduced in LN2666 /generation times close to 25 min and colony formation efficiency $>99 \%$, whatever the insertion; data not shown). Translocation of dif at all of these positions, except $z d d 355$, resulted in a clear Dif ${ }^{-}$phenotype (Table 2, below). Only the insertion of dif at $z d d 355$ restored a Dif ${ }^{+}$phenotype, with generation time and plating efficiency at near wild-type levels, and partial but significant decrease of filament frequency. Thus, dif can fulfill its physiological role when translocated $8 \mathrm{~kb}$ to the right of its natural position but not when it is translocated farther away to its right, or at $37 \mathrm{~kb}$ to the left. The normal competent zone for dif activity is therefore relatively narrow on the chromosome, consistent with the results presented by Kuempel et al. (this issue).

\section{Dif activity does not require termination of replication in its vicinity}

During normal replication, termination takes place at, or to the immediate left of, the pause site $\operatorname{psr} A(=\operatorname{ter} C)$ which is very close to dif (Fig. 1; Louarn et al. 1994). The clockwise moving replication fork probably stalls at psr $A$ for a short while at the end of most replication cycles. To investigate the role of replication fork pausing as a possible elicitor of dif activity, psrA has been removed by a $15-\mathrm{kb}$ deletion to yield strain LN3167. This strain is Dif ${ }^{+}$(Table 3$)$, as are strains having undergone deletions of the tus locus (data not shown), which controls site-specific replication pausing (Hill et al. 1988; Khatri et al. 1989; Lee et al. 1989). In strain LN3034 (Louarn et al. 1994), the region where replication termi-

Table 3. The Dif phenotype in strains modified for the replication fork trap

\begin{tabular}{llcccc}
\hline Strain & Relevant genotype & Filaments & $\begin{array}{l}\text { Doubling } \\
\text { time (min) }\end{array}$ & $\begin{array}{l}\text { Integration } \\
\text { of pFC9 }\end{array}$ & $\begin{array}{l}\text { Dif } \\
\text { phenotype }\end{array}$ \\
\hline LN2666 & $1+1$ & A & 25 & $6.410^{-2}$ & $7.810^{-2}$ \\
LN3167 & $\Delta(z d d 355-z d d 370) \Omega \mathrm{Kn}$ & $\mathrm{A}$ & 26 & $7.110^{-2}$ & + \\
LN3034 & $\Delta(1398-1402) \Omega p s r A^{*}-\mathrm{Ap}$ & $\mathrm{A}$ & 27 & $2.710^{-5}$ & + \\
LN3085 & LN3034 $\Delta($ dif2600)::tet & F & 35 & - \\
\hline
\end{tabular}

Same legend as for Table 2. The construction of strain LN3034 has been described elsewhere (Louarn et al. 1994); ordinates refer to the endpoints of the chromosomal segment substituted with the additional replication terminator. LN3085 is a derivative of LN3034 constructed by transducing the Tet $^{\mathrm{r}}{ }^{\text {determinant }}$ of strain LN3079 (Table 2). 
Table 4. Effect of deletions of the competent zone on dif activity

\begin{tabular}{|c|c|c|c|c|c|c|}
\hline Strain & Relevant genotype & Filaments & $\begin{array}{l}\text { Doubling time } \\
|\mathrm{min}|\end{array}$ & $\begin{array}{l}\text { Efficacy of } \\
\text { plating }(\%)\end{array}$ & $\begin{array}{l}\text { Integration } \\
\text { of pFC9 }\end{array}$ & $\begin{array}{l}\text { Dif } \\
\text { phenotype }\end{array}$ \\
\hline LN2666 & $1+1$ & A & 25 & $>99$ & $6.410^{-2}$ & + \\
\hline LN3073 & $\Delta(z d c 235-z d f 327)::$ tet & $\mathrm{F}$ & 45 & 70 & $1.510^{-4}$ & - \\
\hline LN3076 & $\Delta(z d c 235-z d f 237):$ tet $\Omega$ dif & A & 40 & 94 & $1.510^{-1}$ & + \\
\hline LN3359 & $\Delta(z d c 310-z d d 370)::$ tet & $\mathrm{F}$ & 34 & 68 & $2.210^{-5}$ & - \\
\hline LN3363 & $\Delta(z d c 310-z d d 370)::$ tet $\Omega$ dif & A & 28 & 95 & $5.710^{-2}$ & + \\
\hline LN3074 & $\Delta($ dif-zde381)::tet $\Omega$ dif & A & 28 & 95 & $1.210^{-1}$ & + \\
\hline LN3215 & $\begin{array}{l}\Delta(\text { dif } 2600) \Omega A p \\
\Delta(z d d 355-z d d 370):: \text { tet } \Omega d i f\end{array}$ & $\mathrm{R}$ & 27 & 94 & $7.810^{-2}$ & + \\
\hline LN3169 & $\begin{array}{l}\Delta(\text { dif2600) } \Omega \mathrm{Ap} \\
\Delta(z d d 355-z d d 370 \mid \Omega \mathrm{Kn} \\
z d e 381:: \operatorname{Tn} 10 \Omega d i f\end{array}$ & $\mathrm{~F}$ & 38 & 66 & $1.110^{-1}$ & - \\
\hline
\end{tabular}

Same legend as for Table 2. Strains LN3073 and LN3076 are derived from PK2035 (see Materials and methods). These strains grow poorly even when rendered Dif ${ }^{+}$. This is probably a consequence of the large deletion eliminating useful loci in addition to dif.

nates has been displaced to a position $\sim 200 \mathrm{~kb}$ from dif by inserting an additional pause site. LN3034 is phenotypically Dif ${ }^{+}$(Table 3$)$, and its $\Delta($ dif2600)::tet counterpart displays a Dif ${ }^{-}$phenotype (strain LN3085, Table 3). Thus, the ability of the dif site to fulfill its role in the cell cycle does not require that termination of replication occur in the immediate vicinity of the site.

\section{Dif is active even if replicated early}

Strain LN850 (Louarn et al. 1985) carries a large chromosomal inversion attributable to recombination between two inverted copies of IS5, IS5F at $29 \mathrm{~min}$ (1404 kb on Rudd's map) and IS5T at $78 \mathrm{~min}$ (3674 kb). In this strain, the dif site lies $11 \mathrm{~min}$ from oriC (Fig. 2). Although the inversion is relatively harmless per se, strain LN850 displays a rich medium sensitivity (Rms) caused by the

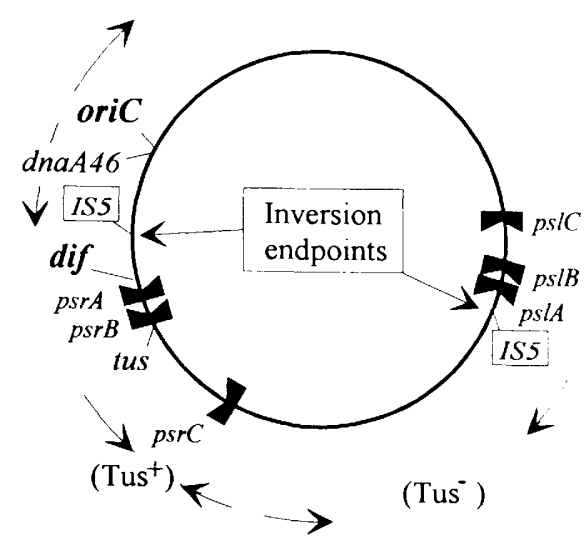

Figure 2. The large Inv $(29-78)$ inversion. This simplified map of the $E$. coli chromosome gives the positions of the endpoints of the rearrangement (the two IS5s) and illustrates its major consequences for chromosome replication: new location of dif close to oriC, and reorientation of the replication terminator sites. Convergent arrows indicate the probable zones of replication fork meeting in Tus ${ }^{+}(\sim 50 \mathrm{~min})$ and Tus ${ }^{-}$conditions (not known precisely). presence of the temperature-sensitive dnaA46 mutation (why the addition of these two genetic alterations results in a $\mathrm{Rms}$ phenotype is not known). The Rms phenotype is nevertheless a convenient indicator of the presence of the rather unstable rearrangement (spontaneous Rmr derivatives are found at frequencies above $\left.10^{-3}\right)$. Previous analyses have shown that the replication arms are very unequal in this strain, because terminators that normally inhibit forks moving clockwise $\operatorname{psr} A=\operatorname{ter} C$, $\operatorname{psr} B=\operatorname{ter} A, \operatorname{psr} C=\operatorname{ter} F \mid$ now inhibit forks moving counterclockwise after a short transit from oriC and early replication of dif (Fig. 2), so that the meeting of forks moving in opposite directions occurs around position 50 min on the map in the Tus ${ }^{+}$conditions, in the vicinity of $\operatorname{psr} C$ (de Massy et al. 1987). In this strain dif is replicated in the first $15 \%$ of a replication cycle, which is $\sim 1.5$ times longer than normal. Because the counterclockwise moving forks pause for a while at $\operatorname{psr} A$, in the immediate vicinity of dif (de Massy et al. 1987), the possibility that fork arrest elicits dif activity can still be considered.

Exponentially growing LN850 bacteria display no filamentation (Fig. 3A) or any sign of SOS induction /actually, the amount of RecA protein per cell is about onethird the normal level; P. Gamas and J.-M. Louarn, unpubl.). Strain LN3213 is derived from LN850 by introduction, through phage P1-mediated transduction of the $J$ (dif2600)::tet deletion. This strain grows very poorly, remains Rms (but Rmr revertants, probably attributable to the restoration of the normal gene order, take over even more rapidly than in LN850), and is strongly prone to filamentation (Fig. 3B).

A $\Delta$ (tus)::Ap ${ }^{\mathrm{r}}$ mutation (Louarn et al. 1994) was introduced into LN850 by transduction to yield strain LN3214, in which the absence of tus sequences was verified by PCR (data not shown). Chromosome replication was analyzed by marker frequency measurements in LN3214. The data (not shown) indicated no evidence for fork arrest at any of the psr sites. Although the inverted part of the chromosome appeared to be replicated at a reduced velocity in the direction opposite to normal, the 


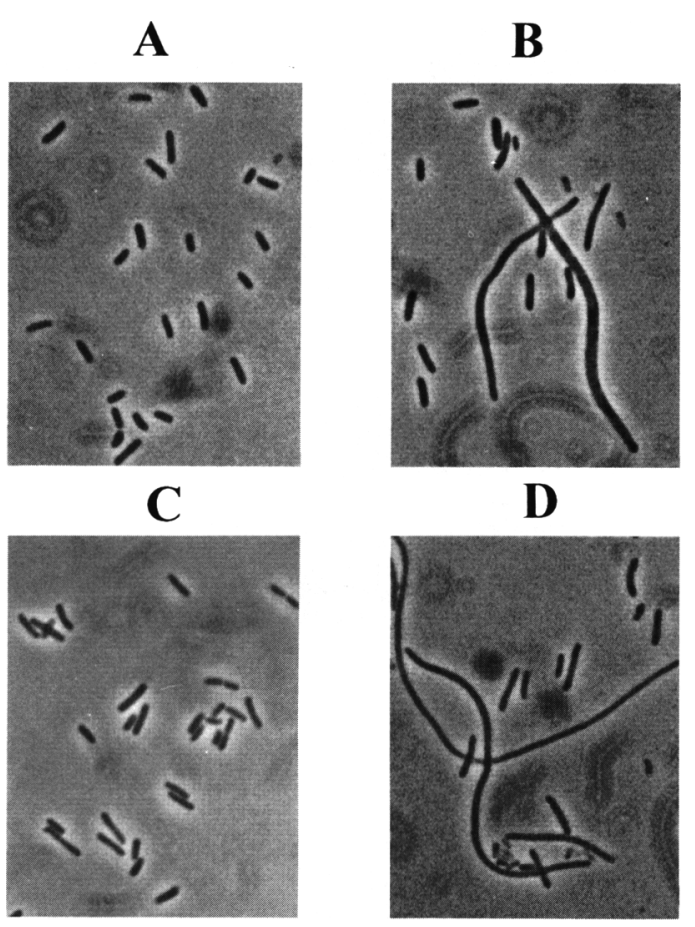

Figure 3. Effect of the deletion of dif on bacteria carrying the large inversion. Bacteria growing exponentially in Vogel and Bonner $\mathrm{E}$ minimal medium at $30^{\circ} \mathrm{C}$ were observed under phasecontrast microscopy with a Leitz Ortholux microscope and photographed. Original magnification, 2300×. $|A|$ LN850 (Inv2978); (B) LN3213 (Inv29-78 $\Delta d i f) ;(C)$ LN3214 (Inv29-78 Jtus); (D) LN3224 (Inv29-78 $\Delta$ dif $\Delta$ tus).

meeting of forks moving in opposite directions occurred at a great distance from dif, $\sim 1 \mathrm{Mb}$ away. No particular tendency to filamentation was detected (Fig. 3C) nor was there any correction of the Rms phenotype. The triple mutant Inv(29-78) $\Delta$ (dif2600)::tet $\Delta($ tus $):: \mathrm{Ap}^{\mathrm{r}}$ (LN3224) behaved similarly to LN3213, showing extensive filamentation (Fig. 3D) and reduced colony-forming ability.

Obviously, dif fulfills its physiological role in the inverted strains, even when termination occurs far away from it. Not only do these data definitely rule out the possibility that termination of replication is an enhancer of dif activity, but they also indicate that the timing of dif replication is unimportant for its physiological activity.

\section{dif activity is independent of any sequence} in the normal competent zone

The regional control over dif activity could be attributable to specific elicitor sequences located within the competence zone. If this were true, the reinsertion of a dif locus into chromosomes deleted for the dif competence region should never give rise to $\mathrm{Dif}^{+}$strains. Two large deletions of the dif region have been assayed (Table 4), a 60-kb deletion between positions $z d c 310$ and $z d d 370$ with a tet fragment joining the deletion endpoints (strain LN3359), and a 155 -kb deletion between positions $z d c 235$ and $z d f 237$ eliminating $57 \mathrm{~kb}$ to the left of dif and $98 \mathrm{~kb}$ to its right with a Tn10 joining the deletion endpoints (strain LN3073; Kuempel et al. 1991). Both strains are $\Delta(d i f)$ and form filaments at high frequency. When the dif-containing fragment was inserted into the tet or Tn10 sequences istrains LN3363 and LN3076), filaments disappeared and doubling times became lower than those of the parent strains (Table 4). Moreover, the efficiency of colony formation was restored to normal (Table 4). These bacteria clearly display a Dif ${ }^{+}$phenotype. These experiments lead to two important conclusions: (1) Inactive dif sequences at positions on either side of the competent zone can become active provided the intervening DNA (i.e., the competent region itself) is deleted; and (2) the competent region contains no unique sequence required for the activity of the translocated sites.

\section{A translocated inactive dif site can be activated by nearby deletions}

Consistent with the above observations, we observed repeatedly that deletion of the region separating an inactive translocated dif site from its natural position results in a Dif ${ }^{+}$phenotype. This was found, for instance, for dif translocations at positions $z d e 381$ (strain LN3074 in Table 4 ) and $z$ de 395 (data not shown), suggesting that such deletions removed determinants rendering the translocated site inactive. To analyze this phenomenon of activation by deletion, we concentrated on the region between $z d d 355$ and $z d d 370$ tet insertions. The presence of dif at position $z d d 370$ (strain LN3162 in Table 2) does not suppress the effects of the $\Delta\left(\right.$ dif2600):: $\mathrm{Ap}^{\mathrm{r}}$ deletion, whereas its presence at position $z d d 355$ (strain LN3161 in Table $2 /$ is suppressive. We deleted the material between the two tet insertions and inserted dif within the tet sequence tagging this $14.7-\mathrm{kb}$ deletion. The resulting strain (LN3215 in Table 4) appeared Dif ${ }^{+}$to the same extent as LN3161 (dif at zdd355, Table 2). Thus, the nonfunctionality of $d i f$ inserted at $z d d 370$ is determined by sequences of the $z d d 355-z d d 370$ interval. Activation of $d i f$ by this deletion is only a local effect. When the $z d d 355-z d d 370$ region was deleted from chromosomes harboring dif at the $z d e 381:: \operatorname{Tn} 10$ position, the resulting strain (LN3169 in Table 4) remained Dif ${ }^{-}$, although the very same translocated dif site was activated by the larger (dif-zde381) deletion (strain LN3074 in Table 4).

\section{Deletion analysis of the transition from inside to outside the competent zone}

One possible explanation for the above results is that specialized border sequences act as barriers separating the zone competent for dif activity from the rest of the chromosome. In this view, strain LN3215 (Table 3; Fig. $4 \mathrm{~A})$, which is $\Delta(z d d 355-z d d 370)::$ tet $\Omega$ dif, is $\mathrm{Dif}^{+}$because the deletion of the $z d d 355-z d d 370$ interval had 


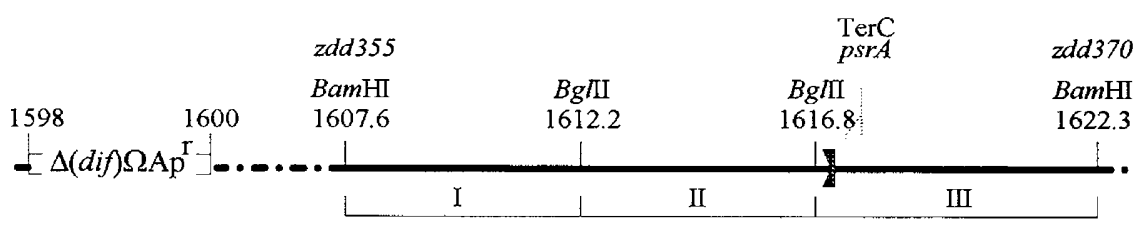

Figure 4. The search for a fixed limit to the DAZ. Presented are the effects of various deletions in the $z d d 355-z d d 370$ region (1607.6-1622.3 kb on Ecomap6; K. Rudd, pers. comm.) on the physiological activity of translocated dif sites. (A) Physical map of the $z d d 355-z d d 370$ region, showing the positions of the BamHI and BglII restriction sites used for creating the rearrangements, and the natural position of dif. $(B)$ The various deletions and their growth characteristics. Segments remaining to the left of the translocated dif site are numbered with reference to $A$. These constructions were created and inserted into the chromosome, as described in Materials and methods. Plasmids (Table 1) involved as intermediates in the constructions: pFC97 for LN3219; pFC95 for LN3220; pFCl03 for LN3286; pFC102 for LN3288; and pFC101 for LN3290.

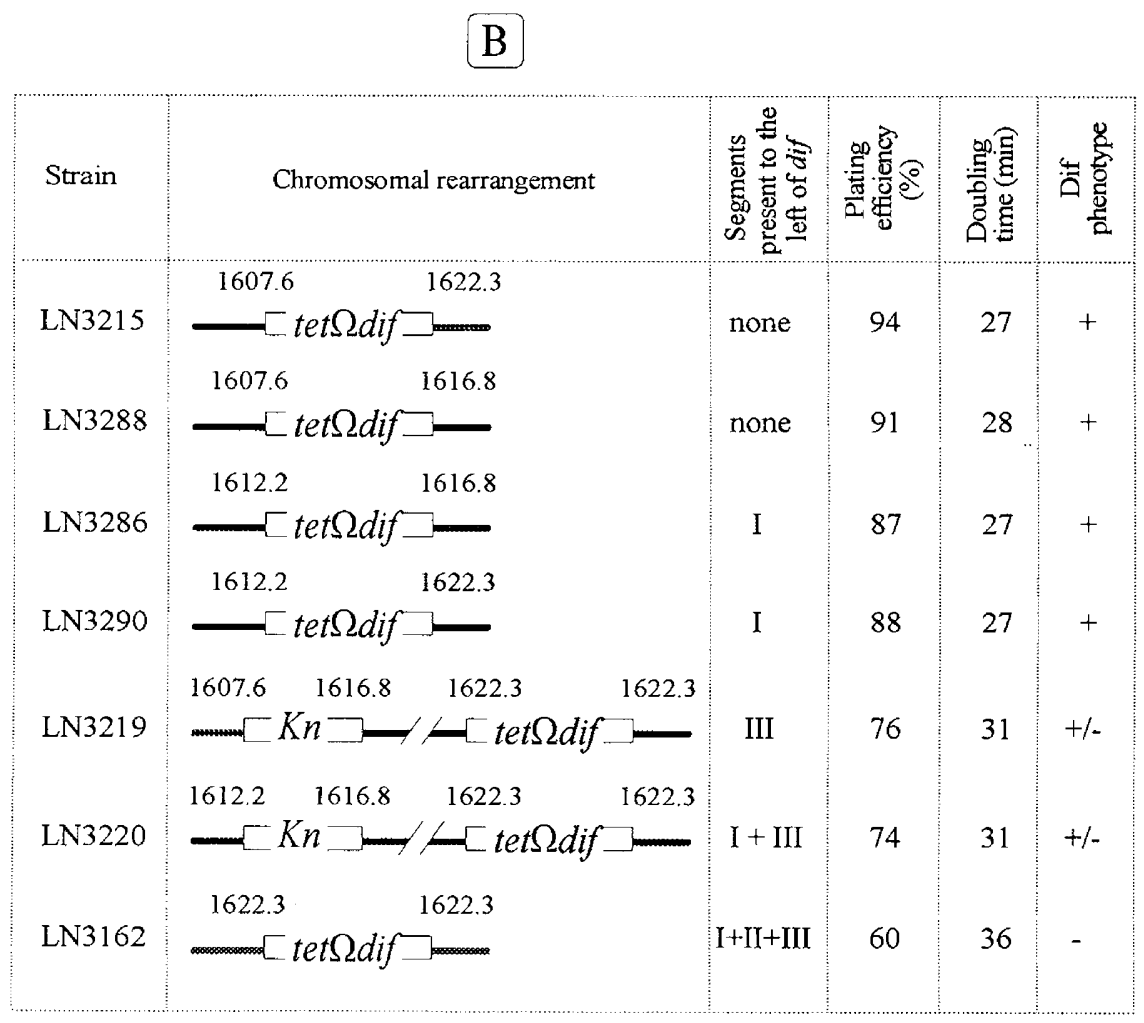

eliminated the right (in reference to Fig. 1) border of the dif activity domain, thus allowing an extension of this domain to $z d d 370$ and beyond. To determine more precisely the map position of the border, partial deletions of the $z d d 355-z d d 370$ interval have been constructed, with replacement of the excised fragment by the tet segment or a $\mathbf{K n}^{\mathrm{r}}$ segment. The deleted segments are numbered I-III in Figure 4A. All strains presented in Figure 4B carried a $\Delta\left(\right.$ dif 2600)::Ap ${ }^{\mathrm{r}}$ deletion plus a dif site translocated either into the tet fragment tagging at the deletion or at the $z d d 370:: \operatorname{Tn} 10$ position. The $\mathrm{Dif}^{+}$phenotype of strains LN3286 and LN3290, in which the dif site is located to the immediate right of segment $\mathrm{I}$, indicates that the region of dif activity extends at least $12 \mathrm{~kb}$ to the right of the natural position of dif. Therefore, the transition from inside to outside the competent zone must occur within segment II or III. Deletion of segment II /or of I + II) when dif is inserted at position $z d d 370$ (strains LN3220 and LN3219) allows some improvement of growth parameters compared with the situation in the undeleted strain strain LN3162 (see also Table 2) but not to the level observed when the whole region is deleted (strain LN3215, see also Table 4). This suggests that segment III also contributes to the lack of functionality of dif at $z d d 370$ when the whole region is present. One possible explanation could be that the hypothetical right barrier limiting the dif activity zone (DAZ) is fully set up only when segments II and III are present. The observation that $d i f$ can display a partial activity $\left(\mathrm{Dif}^{+1-}\right.$ phenotype) was a surprise. Nevertheless, these preliminary data are compatible with the proposed existence of specialized border sequences, even though they raise the possibility that the barrier effect involves rather complicated structures.

\section{Discussion}

Our data, combined with information published previously (Leslie and Sherratt 1995; Tecklenburg et al. 1995) and that presented in Kuempel et al. (this issue), confirm 
that the physiological functions of dif can be achieved only when the site is situated within a narrow zone perhaps $20-30 \mathrm{~kb}$ ) around its natural position, even though it is able to recombine with a plasmid-borne dif site whatever its chromosomal location. This narrow competent zone is termed DAZ in the following. The main features of this position effect can be summarized as follows (1) The replication process is not directly involved. Although dif is normally present in the region where replication terminates, its activity is unrelated to termination of chromosome replication and does not require that the site is replicated at a precise time during the cell cycle. (2) The genetic content of the region competent for dif physiological activity is unimportant. Once the normal region competent for dif activity is deleted, a new competent region takes the place of the old one, implying that the competence is determined by factors external to the zone. (3) Deletion of the transition region between the DAZ and the noncompetent adjacent region does not inhibit the regional control over dif activity but, rather, seems to allow the extension of this control to positions normally incompetent. The transition region is therefore not an elicitor of competence but, rather, a barrier to propagation of competence. The fact that competence remains restricted to a rather small domain when a deletion has modified the original DAZ is suggestive of a plurality of sequences having the same potential as the transition region. (4) The transition from inside the domain of competence to outside on the right of the DAZ seems to involve specialized sequences. Dissection and reconstruction experiments are aimed at identifying these specialized sequences and should provide a more complete view of the transition region.

Strikingly, the observations and rules established on the DAZ also hold for terminal recombination (Louarn et al. 1994). The normal TRZ (terminal recombination zone) occupies a small fraction of the chromosome that overlaps the DAZ and is susceptible to the same kinds of chromosome rearrangements. For instance, strains deleted for the normal TRZ still display excisive hyperrecombination at the junction of the deleted segment, and termination of chromosome replication does not appear to be a controlling factor (Louarn et al. 1994). Terminal recombination is dependent on $\operatorname{Rec} B C(D)$, a fact implying that, in the experimental system we use, terminal recombination is an interchromosomal event (J. Louarn, F. Cornet, J. Patte, and J.M. Louarn, unpubl.). It also displays a polarization on both sides of the DAZ, which is consistent with a frequency of initiation events increasing gradually on both sides of the DAZ and culminating within this region (J. Louarn, F. Cornet, J. Patte, and J.M. Louarn, unpubl.).

How can we explain the restriction of dif activity to a narrow zone of competence, and what is the phenomenon that, occurring preferentially within the DAZ but not depending on unique sequences in this zone, facilitates terminal recombination and makes dif-specific recombination physiologically active? Localized intercatenation of sister chromosomes, which could in turn favor local interchromosomal recombination, is an obvi- ous candidate. Surprisingly, termination of replication, which has been proven to favor intercatenation in some systems such as SV40 replication (Sundin and Varshavsky 1981), is not an elicitor of either dif activity (this work) or terminal recombination (Louarn et al. 1994), although both phenomena occur in the normal termination region. The model proposed for terminal recombination involved nucleoid organization (Louarn et al. 1994). We postulated that nucleoid reconstruction after replication occurs in the same direction as normal replication, in a sequential process involving specialized and ordered sequences. The model implied the existence of two polarized organization units along each replication arm, with a region (named the nucleoid terminus) separating these two units near the terminus. It might be that catenation links are progressively chased and accumulate in the nucleoid terminus as nucleoid reconstruction proceeds along the two still intercatenated sister chromosomes. Associated with decatenation or otherwise, interchromosomal exchanges by homologous recombination would be favored in the region, which can be considered as a zone of synapsis between sister chromosomes. This model can also provide the framework for an explanation of the restricted zone competent for dif activity. In this view, the DAZ is the nucleoid terminus, and the sequences involved in the transition between the DAZ and the rest of the chromosome should be the last organizing sequences of each organization unit. Also, a multiplicity of sequences involved in structuring the nucleoid makes it simple to envisage the creation of a new competent zone for dif activity after deletion of the original one. There must always be a zone where catenation links accumulate between the two rearranged organization units.

Interestingly, the existence of a fixed and polarized nucleoid organization has already been proposed to explain the seemingly unrelated observation that regions flanking the terminus (and spanning $20 \%-30 \%$ of the chromosome) are refractory to genetic inversions [non divisible zones (NDZ)s; Rebollo et al. 1988]. Recent explorations (M. Guijo, V. François, J. Patte, J.M. Louarn, and J.E. Rebollo, in prep.) have revealed that the NDZs are not continuous but rather that they encompass a series of domains, each refractory to inversion. We suspect that these domains are involved in the process of polarized nucleoid organization that we postulate here, and it may appear in the future that the DAZ, the TRZ, and the zones refractory to inversion $(\mathrm{NDZ})$ are created by the same features of nucleoid organization. In the case of the large 29-78 inversion used in this work, one endpoint, the IS5F at $29 \mathrm{~min}$, is probably located in a region linking two inversion-refractory domains, so that the crucial features of nucleoid organization might have been preserved after inversion, even though this $10-$ to $20-\mathrm{kb}$ linking region has been replaced by a $2000-\mathrm{kb}$ one. This should not modify the postulated process of catenation link migration, at least along the chromosomal arm that has conserved its normal organization. Hence, the region competent for dif activity would not be expected to be modified by the enormous chromosome rearrangement. 


\section{Materials and methods}

Strains and plasmids

Plasmids used and their constructions are presented in Table 1. All strains derived from LN2666, a spontaneous streptomycinresistant mutant of $\mathrm{CB} 0129\left(\mathrm{~F}^{-} \mathrm{W} 1485\right.$ leu thy $A$ deoB or $C$ supE; Bird et al. 1972), except LN3073, which is a spontaneous streptomycin-resistant mutant of PK2035 (Kuempel et al. 1991), and LN850, which is derived differently from CBO129 (Louarn et al. 1985). LN3079 and LN3061 are LN2666 derivatives made $\Delta\left(\right.$ dif2600)::tet and $\Delta\left(\right.$ dif2600)::Ap ${ }^{\mathrm{x}}$ by homologous recombination with plasmid pFC75.1 and pFC73, respectively (integration then excision). The zde381::Tn10, zde395::Tn10, and zde406::Tn10 insertions from strains LN2035, LN1954, and LN1953 (Louarn et al. 1994) were transferred by phage Pl-mediated transduction into LN3061. The $\Delta \mid z d c 310$-zdd370|::tet and $\Delta($ dif-zde381)::tet deletions were created by homologous recombination using the "tes and tek" system (François et al. 1987).

\section{Media and general procedures}

All in vivo experiments were carried out in LB medium (Miller 1992), except for LN850 and its derivatives, which were grown in $\mathrm{M} 9$ glucose medium or Vogel and Bonner E minimal medium as described previously (Louarn et al. 1985). Antibiotic concentrations used were $200 \mu \mathrm{g} / \mathrm{ml}$ of streptomycin, $20 \mu \mathrm{g} / \mathrm{ml}$ of chloramphenicol, $10 \mu \mathrm{g} / \mathrm{ml}$ of tetracyclin, $50 \mu \mathrm{g} / \mathrm{ml}$ of ampicillin, $50 \mu \mathrm{g} / \mathrm{ml}$ of spectinomycin, and $25 \mu \mathrm{g} / \mathrm{ml}$ of kanamycin. All plasmid constructions involved standard procedures (Sambrook et al. 1989; Table 1).

\section{Doubling time and plating efficiency measurements}

Generation times during exponential growth were determined on bacteria incubated in LB medium at $37^{\circ} \mathrm{C}$ with vigorous agitation for at least 4-6 hr and diluted into prewarmed LB medium to keep $\mathrm{OD}_{540} \mathrm{~nm}$ below 0.2 at initial measurement. Plating efficiency was monitored by phase-contrast examination, at magnification $400 \times$ with a Nikon Labophot-2A microscope, of bacteria growing on microscope slides spread with $0.6 \mathrm{ml}$ of soft LB medium (Miller 1992), after incubation for $3 \mathrm{hr}$ at $37^{\circ} \mathrm{C}$ in a wet chamber. The fate of at least 500 individual cells was followed for each determination. Plating efficiency is expressed as the percent of spread bacteria giving rise to microcolonies. Measuring the dynamic equilibrium between viable and inviable bacteria in an expanding population, this test gives reproducible results if growth conditions, in particular aeration, are kept as standardized as possible before spreading the bacteria on the slide.

\section{Test for the presence of dif on the chromosome}

The presence and functionality of a translocated dif site was tested by determining the efficiency of rescuing of plasmid pFC9 at $42^{\circ} \mathrm{C}$, a dif-carrier replication-thermosensitive pSC101 derivative, as described previously (Cornet et al. 1994). Integration frequencies given in Tables 2, 3, and 4 are the average of four independent measurements.

\section{Precise substitution of chromosomal segments}

Use of plasmids of the pFCl3 family for substitution of chromosomal segments had been described previously /Cornet et al. 1994). Chromosomal fragments are first cloned in pFC13-derived vectors and then modified to get the desired construction (see Table 1). Transfer into the chromosome of the various constructions was performed by a two-step procedure. First, plasmid integration into the chromosome is selected by plating on chloramphenicol-containing medium at $42^{\circ} \mathrm{C}$; second, plasmid excision and loss are selected by plating on streptomycin-containing medium at $42^{\circ} \mathrm{C}$. The cured colonies are then screened for the desired antibiotic resistances. For example, dif translocations involved use of plasmids pFC72.1 and pFC72.2, which carry the $\Omega$ dif fragment inserted into the $\operatorname{Tn} 10$ tet $A$ gene in either orientation (Fig. 1), and the success of transfer of dif into prexisting chromosomal $\operatorname{Tn} 10$ or tet insertions was monitored by screening $\mathrm{Sm}^{\mathrm{r}}$ derivatives for loss of tetracyclin resistance.

\section{In vivo cloning of the $z \mathrm{dd} 355-\mathrm{zdd} 370$ region}

We took advantage of plasmid pFC92, which was constructed in vitro and carries the regions left of $z d d 355$ and right of $z d d 370$ joined by a $\mathrm{Kn}^{\mathrm{r}}$ interposon (Table 1). LN2666 bacteria carrying this plasmid integrated into the chromosome $\left(\mathrm{Cm}^{\mathrm{T}}\right.$ at $\left.42^{\circ} \mathrm{C}\right\}$ were grown at $42^{\circ} \mathrm{C}$ in LB medium without antibiotics, and the rare plasmid molecules liberated by excision from the chromosome were prepared by standard alkaline lysis technique. Among the excision events, those taking place in the region not used for integration should result in plasmids harboring the wild-type $z d d 355-z d d 370$ region. The plasmid preparation was used to transform strain LN2521 [CB0129 د(tus) $\Omega A$; Louarn et al. 1994], with selection for resistance to chloramphenicol and then screening for kanamycin sensitivity. Among $67 \mathrm{Cm}^{\mathrm{r}}$ transformants, 4 were $\mathrm{Kn}^{\text {s }}$ and all of these harbored the desired plasmid, pFC94, which carries the 1602- to $1624-\mathrm{kb}$ chromosomal segment.

\section{Construction of deletions in the $z \mathrm{dd} 355-\mathrm{zdd} 370$ region}

Various segments, numbered I-III in Figure 4A, have been deleted from the pFC94 plasmid by partial digestions with BamHI or $B g / \mathrm{II}$ and replaced by either the tet segment or a $\mathrm{Kn}^{\mathrm{r}}$ determinant. The tagged deletions have been eventually inserted into the chromosome, as described above. To ascertain the presence in the chromosome of modified $z d d 355-z d d 370$ regions, these regions were picked up from the chromosome as described in the above section, except that the sequence picker was plasmid pFC91, which carries the untagged $د(z d d 355-z d d 370)$ deletion (Table 1). Because all rearranged strains carried a tet fragment inserted within the $z d d 355-z d d 370$ region (Figs. 3 and 5), the in vivo recombinant plasmids could be directly selected for resistance to tetracyclin. Restriction maps of these plasmids were eventually determined. In all cases, they corresponded to the predicted structures of the various rearrangements. The ancestors (before insertion of the $\Omega$ dif fragment) of strains, except LN3162 and LN3288, presented in Figure 4B have been verified by this procedure.

\section{Acknowledgments}

We thank Dr. P. Kuempel for constant exchange of information and discussions, M.L. Bortolin for her participation in some of the experiments, and J.-Y. Bouet and P. Polard for frequent and useful discussions. We are indebted to Dr. D. Lane for his thorough critical reading of the manuscript. This study was supported in part by grants from the Association de la Recherche sur le Cancer (ARC) (contract 6269) and the European Economic Community icontract 9269|. F.C. was supported by an ARC fellowship.

The publication costs of this article were defrayed in part by 
payment of page charges. This article must therefore be hereby marked "advertisement" in accordance with 18 USC section 1734 solely to indicate this fact.

\section{References}

Béjar, S. and J.P. Bouché. 1983. Molecular cloning of the region of the terminus of Escherichia coli K12 DNA replication. /. Bacteriol. 153: 604-609.

Bird, R., J. Louarn, J. Martuscelli, and L. Caro. 1972. Origin and sequence of chromosome replication in Escherichia coli. I. Mol. Biol. 70: 549-566.

Blakely, G., S. Colloms, G. May, M. Burke, and D. Sherratt. 1991. Escherichia coli XerC recombinase is required for chromosomal segregation at cell division. New Biol. 3: 789798.

Blakely, G., G. May, R. McCulloch, L.K. Arciszewska, M. Burke, S.T. Lovett, and D.J. Sherratt. 1993. Two related recombinases are required for site-specific recombination at dif and cer in E. coli $\mathrm{K} 12$. Cell 75: 351-361

Cornet, F., I. Mortier, J. Patte, and J.-M. Louarn. 1994. Plasmid pSC101 harbors a recombination site, psi, which is able to resolve plasmid multimers and to substitute for the analogous chromosomal E. coli site, dif. I. Bacteriol. 176: $3188-$ 3195.

de Massy, B., S. Béjar, J. Louarn, J.-M. Louarn, and J.-P. Bouché. 1987. Inhibition of replication forks exiting the terminus region of the Escherichia coli chromosome occurs at two loci separated by 5 min. Proc. Natl. Acad. Sci. 84: 1749-1963.

Fellay, R., J. Frey, and H.K. Krisch. 1987. Interposon mutagenesis of soil and water bacteria: A family of DNA fragments designed for in vitro insertional mutagenesis of Gram-negative bacteria. Gene 52: 147-154.

François, V., J. Louarn, J. Patte, and J.-M. Louarn. 1987. A system for in vivo selection of genomic rearrangements with predetermined endpoints in Escherichia coli using modified Tn 10 transposons. Gene 56: 99-108.

Hill, T.M., A.J. Pelletier, M. Tecklenburgh, and P.L. Kuempel. 1988. Identification of a DNA sequence from the $E$. coli terminus that halts replication forks. Cell 55: 459-466.

Khatri, G.S., T. MacAllister, P.R. Sistra, and D. Bastia. 1989 The replication terminator protein of $E$. coli is a DNA sequence-specific contra-helicase. Cell 59: 667-674.

Kohara, Y.K., A. Akiyama, and K. Isono. 1987. The physical map of the whole Escherichia coli chromosome: Application of a new strategy for rapid analysis and sorting of large genomic libraries. Cell 50: 495-508.

Kuempel, P.L., J.M. Henson, L. Dircks, M. Tecklenburg, and D.F. Lim. 1991. dif, a recA-independent recombination site in the terminus region of Escherichia coli. New Biologist 3: 799-811.

Kuempel, P.L., A. Hogaard, M. Nielsen, O. Nagapan, and M. Tecklenburg. 1996. Use of a transposon Tndif to obtain suppressing and non-suppressing insertions of the dif resolvase site of Escherichia coli. Genes \& Dev. (this issue).

Lee, E.H., A. Kornkerg, M. Hidaka, T. Kobayashi, and T. Horiuchi. 1989. Escherichia coli replication termination protein impedes the action of helicases. Proc. Natl. Acad. Sci. 86: 9104-9108.

Leslie, N.R. and D.J. Sherratt. 1995. Site-specific recombination in the replication terminus region of Escherichia coli: Functional replacement of dif. EMBO J. 14: 1561-1570.

Louarn, J.M., J.P. Bouché, F. Legendre, J. Louarn, and J. Patte. 1985. Characterization and properties of very large inversions of the E. coli chromosome. Mol. \& Gen. Genet.
201: $467-476$.

Louarn, J.-M., J. Louarn, V. François, and J. Patte. 1991. Analysis and possible role of hyperrecombination in the termination region of the Escherichia coli chromosome. I. Bacteriol. 173: 5097-5104.

Louarn, J., F. Cornet, V. François, J. Patte, and J.M. Louarn. 1994. Hyperrecombination in the terminus region of the Escherichia coli chromosome: Possible relation to nucleoid organization. /. Bacteriol. 176: 7524-7531.

Miller, J.H. 1992. A short course in bacterial genetics. Cold Spring Harbor Laboratory Press, Cold Spring Harbor, N.Y.

Rebollo, J.E., V. François, and J.-M. Louarn. 1988. Detection and possible role of two large nondivisible zones on the Escherichia coli chromosome. Proc. Natl. Acad. Sci. 85: 93919395.

Sambrook, J., E.F. Fritsch, and T. Maniatis. 1989. Molecular cloning: A laboratory manual, 2nd ed. Cold Spring Harbor Laboratory Press, Cold Spring Harbor, N.Y.

Sherratt, D.J., L.K. Arciszewska, G. Blakely, S. Colloms, K. Grant, N. Leslic, and R. McCulloch. 1995. Site-specific recombination and circular chromosome segregation. Phil. Trans. R. Soc. Lond. 347: 37-42.

Sundin, O. and A. Varshavsky. 1981. Arrest of segregation leads to accumulation of highly intertwined catenated dimers: Dissection of the final stages of SV40 DNA replication. Cell 25: 659-669.

Tecklenburg, M., A. Naumer, O. Nagappan, and P.L. Kuempel. 1995. The dif resolvase locus of the Escherichia coli chromosome can be replaced by a 33-bp sequence, but function depends on location. Proc. Natl. Acad. Sci. 92: 1352-1356. 


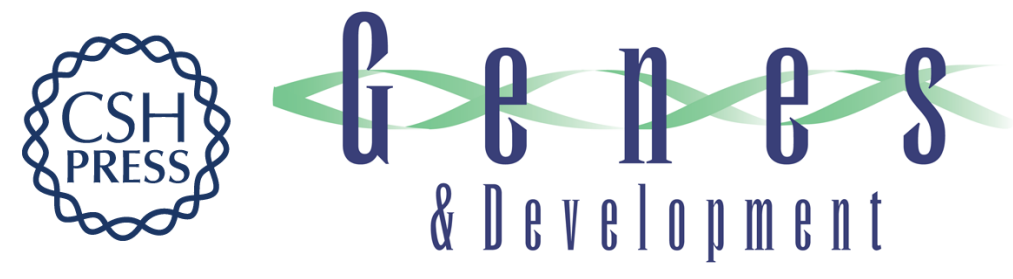

\section{Restriction of the activity of the recombination site dif to a small zone of the Escherichia coli chromosome.}

F Cornet, J Louarn, J Patte, et al.

Genes Dev. 1996, 10:

Access the most recent version at doi:10.1101/gad.10.9.1152

References This article cites 21 articles, 8 of which can be accessed free at:

http://genesdev.cshlp.org/content/10/9/1152.full.html\#ref-list-1

License

Email Alerting

Service

Receive free email alerts when new articles cite this article - sign up in the box at the top right corner of the article or click here.

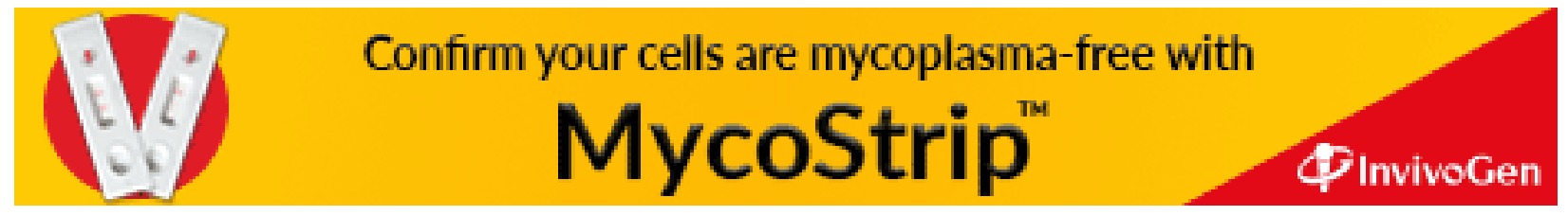

